ARTICLE

https://doi.org/10.1038/s41467-019-12816-z

\title{
Regulation of the ER stress response by a mitochondrial microprotein
}

Qian Chu' ${ }^{1}$, Thomas F. Martinez (1) ${ }^{1}$, Sammy Weiser Novak ${ }^{2}$, Cynthia J. Donaldson ${ }^{1}$, Dan Tan ${ }^{1}$, Joan M. Vaughan ${ }^{1}$

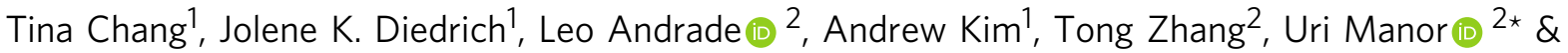
Alan Saghatelian (i) ${ }^{1 *}$

Cellular homeostasis relies on having dedicated and coordinated responses to a variety of stresses. The accumulation of unfolded proteins in the endoplasmic reticulum (ER) is a common stress that triggers a conserved pathway called the unfolded protein response (UPR) that mitigates damage, and dysregulation of UPR underlies several debilitating diseases. Here, we discover that a previously uncharacterized 54-amino acid microprotein PIGBOS regulates UPR. PIGBOS localizes to the mitochondrial outer membrane where it interacts with the ER protein CLCC1 at ER-mitochondria contact sites. Functional studies reveal that the loss of PIGBOS leads to heightened UPR and increased cell death. The characterization of PIGBOS reveals an undiscovered role for a mitochondrial protein, in this case a microprotein, in the regulation of UPR originating in the ER. This study demonstrates microproteins to be an unappreciated class of genes that are critical for inter-organelle communication, homeostasis, and cell survival.

\footnotetext{
${ }^{1}$ The Salk Institute for Biological Studies, Clayton Foundation Laboratories for Peptide Biology, 10010N. Torrey Pines Rd, La Jolla, CA 92037, USA. ${ }^{2}$ The Salk Institute for Biological Studies, Waitt Advanced Biophotonics Center, 10010N. Torrey Pines Rd, La Jolla, CA 92037, USA. *email: umanor@salk.edu; asaghatelian@salk.edu
} 
T he term microproteins refers to peptides and small proteins translated from small open reading frames $(\mathrm{smORFs})^{1,2}$. Advances in genomics and proteomics technologies reveal that mammalian genomes harbor hundreds to thousands of previously unannotated microprotein-coding $\mathrm{smORFs}^{3-5}$. As a large and completely unstudied fraction of the genome, assignment of functions to smORFs and microproteins represents a major opportunity to gain new insights into biology. Only a handful of smORFs and microproteins have been characterized so far $^{1,2}$. For example, several muscle-specific smORFs have revealed new pathways that control muscle performance and development ${ }^{6,7}$, and a microprotein called CYREN regulates DNA repair pathway choice during the cell cycle ${ }^{8}$. It is likely that many other key cellular processes are also mediated by uncharacterized microproteins and the discovery and characterization of smORFs and microproteins is an important research endeavor.

Cells routinely encounter stress that negatively impacts cell health and function. The unfolded protein response (UPR) is a fundamental pathway in eukaryotes that is triggered by the onset of endoplasmic reticulum (ER) stress resulting from the presence of unfolded proteins in the ER lumen ${ }^{9,10}$. Stress-responsive genes, proteins, and pathways provide a cellular mechanism to cope with this stress and return cells to homeostasis. There are three primary branches of the UPR pathway and each pathway is mediated by a different ER protein: IRE1, PERK, or ATF6 ${ }^{11}$. Activation of these proteins during UPR initiates signals at the ER that slow down protein expression, increase protein folding, and upregulate degradation of unfolded proteins $s^{9,10}$. If these steps fail to return the cell to homeostasis and prolong activation of UPR, the cells will undergo apoptosis ${ }^{12,13}$. Understanding UPR regulation has implications for human health as dysregulation of UPR signaling is thought to underlie several prevalent diseases $^{14,15}$. Here, we characterize a microprotein called PIGBOS and reveal a role for a mitochondrial protein in UPR signaling.

\section{Results}

PIGBOS is a conserved microprotein. During proteomic searches for microproteins, we identified a tryptic peptide, MQLVQESEEK, from the human 54-amino acid PIGB opposite strand 1 (PIGBOS) microprotein (Fig. 1a), providing experimental evidence for PIGBOS translation. PIGBOS obtained its name because it is on the opposite strand of the phosphatidylinositol glycan anchor biosynthesis class B (PIGB) gene (Fig. 1b). The PIGBOS transcript consists of two exons and has three splice isoforms with slight differences in the first exon, but the second exon that contains the entire PIGBOS smORF is the same (Supplementary Fig. 1a). RNA-Seq and Ribosome profiling datasets provide evidence of PIGBOS expression and translation in three different human cell lines (Supplementary Fig. 1a). To further confirm whether the PIGBOS smORF is translated to produce a stable microprotein, we raised antibodies against human and rat PIGBOS. Western blot analysis of numerous human cell lines (Supplementary Fig. 1b) and rat tissues (Fig. 1c) readily detected PIGBOS, demonstrating PIGBOS to be a widely expressed, stable microprotein. PIGBOS is uncharacterized, but sequence conservation and a positive PhyloCSF score ${ }^{16}$ suggest that this microprotein is functional (Fig. 1d). PIGBOS has no paralogs or homologs which prevents any molecular, cellular, or physiological function from being inferred, and requires the de novo characterization of PIGBOS.

PIGBOS is a mitochondrial outer membrane (MOM) microprotein. Subcellular localization provides valuable information to assess the function of an uncharacterized protein. PIGBOS was found in the mitochondrial fraction by Western blot and proteomics, but not in any other subcellular fractions tested (Fig. 2a and Supplementary Fig. 2a). We validated PIGBOS's mitochondrial localization by imaging exogenously expressed PIGBOS-FLAG in HeLa cells and immunofluorescence of endogenous PIGBOS in rat C6 cells (we used rat cells because of the superior performance of the rat anti-PIGBOS antibody), which showed puncta that overlap with the mitochondrial marker Tom 20 (Fig. 2b and Supplementary Fig. 2b). Sequence analysis using Transmembrane Helix Prediction (TMHMM) ${ }^{17}$ revealed that PIGBOS is a single-pass transmembrane protein with a transmembrane region between amino acids 7-29 (Fig. 1d). To determine whether PIGBOS is localized to the inner or outer mitochondrial membrane, we used a protease protection assay $^{18,19}$. Proteolysis of isolated mitochondria with proteinase $\mathrm{K}$ under conditions that retain MOM integrity led to the degradation of PIGBOS-an identical result to that of the MOM protein Tom20-indicating that PIGBOS is a MOM microprotein (Fig. 2c).

To validate this result in live cells, we turned to an optimized split GFP approach ${ }^{20}$ whereby three repeats of the last beta-strand of GFP $(3 \times$ GFP11) are fused to the C-terminus of PIGBOS (PIGBOS-3 $\times$ GFP11) and the non-fluorescent remainder of the GFP, i.e., GFP(1-10), is co-expressed. In this system, fluorescence is only observed if GFP11 and GFP(1-10) interact to reconstitute the intact GFP beta-barrel (i.e., GFP11 + GFP(1-10)) ${ }^{20}$. Coexpression of PIGBOS-3 $\times$ GFP11 and the GFP(1-10) resulted in a fluorescent ring around the mitochondria (Fig. 2d and Supplementary Fig. 2c), consistent with the aforementioned biochemical data showing that PIGBOS is localized to the MOM. The same mitochondrial outline has been observed for the MOM protein Tom $20^{21}$. Because the $3 \times$ GFP11 must interact with cytosolic GFP (1-10) to reconstitute a fluorescent GFP, this experiment also suggested that the C-terminus of PIGBOS is cytoplasmic. Placement of $3 \times$ GFP11 at the PIGBOS N-terminus $(3 \times$ GFP11PIGBOS-FLAG) readily localized PIGBOS in the mitochondria but produced no fluorescence because it cannot interact with GFP (1-10) (Supplementary Fig. 2d). PIGBOS's topology indicates that it belongs to a group of MOM proteins called signal-anchor proteins that rely on their transmembrane domains to localize the proteins to the mitochondria and anchor the proteins to the $\mathrm{MOM}^{22}$.

The PIGBOS microprotein interacts with the ER protein CLCC1. Protein interaction studies can accelerate the characterization of microproteins $s^{8,23}$, so we attempted to use this strategy to characterize PIGBOS. Proteomics of immunoprecipitated PIGBOS-FLAG followed by SAINT ${ }^{24}$ and CRAPome ${ }^{25}$ analysis to remove false positives and contaminating proteins identified chloride channel CLIC-like 1 (CLCC1) as a PIGBOS-interacting protein (Fig. 3a, b and Supplementary Data 1 and Supplementary Tables 1 and 2). We validated this interaction in live cells using a proximity labeling assay ${ }^{26}$ with a PIGBOS-engineered ascorbate peroxidase 2 fusion protein (PIGBOS-APEX), which showed robust biotinylation and enrichment of CLCC1, whereas the ER marker Sec61b showed no enrichment and was equally biotinylated by PIGBOS-APEX and APEX control (Fig. 3c, d and Supplementary Fig. 3a). The protein interaction data indicated that PIGBOS and CLCC1 are in close proximity and that PIGBOS specifically interacts with CLCC1. A reciprocal immunoprecipitation assay with an HA-tagged CLCC1 (CLCC1-HA) enriched PIGBOS-FLAG, which further indicated the interaction between the two proteins (Supplementary Fig. 3b). CLCC1 is a putative chloride channel localized to the $\mathrm{ER}^{27}$, though some data suggests it might be found in the nucleus, Golgi, and plasma membrane as well ${ }^{28}$. Due to this ambiguity, we reassessed CLCC1 localization 

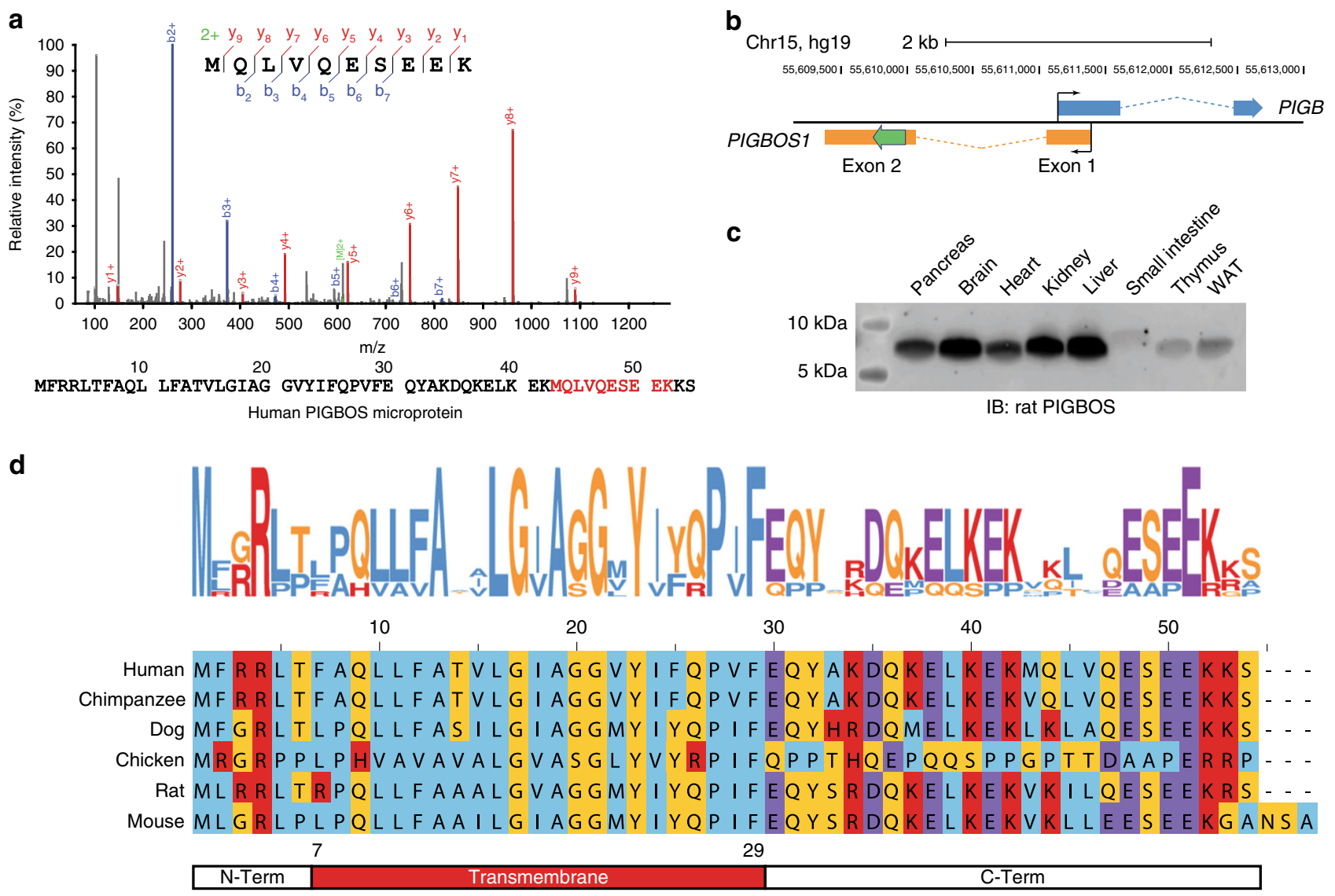

Fig. 1 PIGBOS is a conserved microprotein. a Detection of a unique PIGBOS tryptic peptide by proteomics (MS/MS spectrum as shown) and the entire 54 amino acid human PIGBOS microprotein with detected tryptic peptide (red). b PIGBOS1 gene contains two exons and is located on the opposite strand of the PIGB gene on chromosome 15. The PIGBOS protein coding sequence (CDS) in exon 2 is highlighted in green. c Western blot of rat tissues detects endogenous PIGBOS microprotein expression. $\mathbf{d}$ A logo plot generated from the sequence alignment of PIGBOS microprotein from multiple species reveals conserved amino acids including the transmembrane region between amino acids 7-29 (red) flanked by a N-terminal region (N-Term, aa 1-6) and a Cterminal region (C-Term, aa 30-54)

by imaging CLCC1-HA and the ER marker Sec61b-mCherry, which showed substantial overlap (Supplementary Fig. 4a). By contrast, counterstaining of CLCC1-HA and the Golgi marker GM130 revealed no colocalization (Supplementary Fig. 4b). Together, these experiments indicated that CLCC1 is primarily an ER protein.

The PIGBOS C-terminus is required for the CLCC1 interaction. Based on PIGBOS's topology, the cytosolic C-terminus of PIGBOS should mediate the interaction with CLCC1; however, testing this model by simply removing the entire PIGBOS Cterminal region (amino acids 30-54) was unsuccessful because the truncated PIGBOS(1-29)-FLAG showed no detectable expression. Instead, we swapped the 25 -amino acid PIGBOS Cterminal region with the three GFP11 repeats (PIGBOS- $\Delta \mathrm{C}-3 \times$ GFP11-FLAG). This PIGBOS variant has robust mitochondrial localization (Supplementary Fig. 5) but does not enrich CLCC1 after immunoprecipitation (Fig. 3e), demonstrating the requirement of the C-terminal region for an interaction with CLCC1. In an attempt to define the specific amino acids needed for binding, we mutated blocks of three consecutive amino acids of the Cterminal region to alanine (Supplementary Fig. 6a). Confocal imaging showed that all of the triple alanine PIGBOS- $3 \times$ GFP11FLAG mutants were expressed and localized to mitochondria (Supplementary Fig. 6b). Immunoprecipitation experiments identified amino acids $30-36$ of PIGBOS to be critical for CLCC1 binding since mutation of these amino acids to alanine resulted in decreased CLCC1 enrichment (Supplementary Fig. 6c). In total, these results demonstrated that the $\mathrm{C}$-terminal region of PIGBOS is essential for the PIGBOS-CLCC1 interaction.

Measuring the interaction between PIGBOS and CLCC1 in cells. We next used a split-GFP bimolecular complementation strategy to image and quantify the PIGBOS-CLCC1 interaction. Co-expression of PIGBOS-3 $\times$ GFP11 and CLCC1-GFP(1-10) resulted in a robust green fluorescent signal, whereas the $\mathrm{C}$ terminus truncated PIGBOS- $\Delta \mathrm{C}-3 \times$ GFP11-FLAG failed to fluoresce (Fig. 4a, b). This result indicated that the reconstituted GFP fluorescence depends on PIGBOS-CLCC1 interaction, making this an ideal assay to measure the PIGBOS-CLCC1 interaction in cells. Overlap of the GFP and PIGBOS fluorescence signals indicated that not all PIGBOS is interacting with CLCC1 (Fig. 4b). In addition, spatial analysis of the fluorescence distribution revealed that the GFP signal peaks between the fluorescent reporter signals for the MOM (Tom20) and ER (Sec61bmCherry), providing further evidence that the PIGBOS-CLCC1 interaction occurs at the ER-mitochondria interface (Fig. 4a).

To assess the PIGBOS-CLCC1 interaction in bulk and in live cells, we developed a flow cytometry experiment to measure the amount of reconstituted GFP ${ }^{29}$. Cells expressing PIGBOS-3 $\times$ GFP11 and CLCC1-GFP(1-10) had a robust GFP signal with $\sim 30 \%$ of the cellular population above the minimum threshold and significantly higher mean GFP intensity compared to mock transfected controls. By contrast, the C-terminus truncated 
a

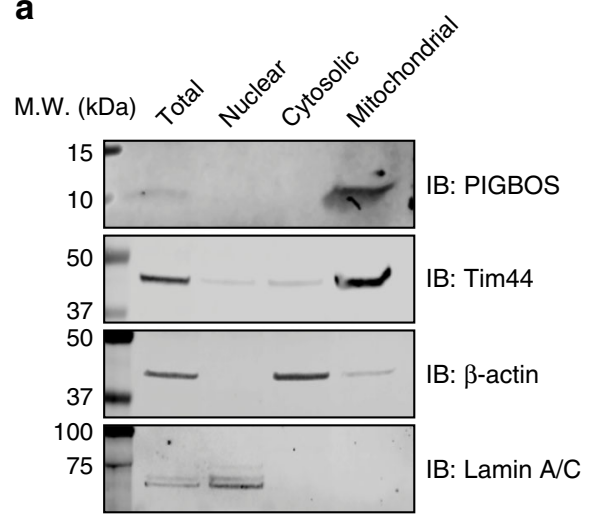

C

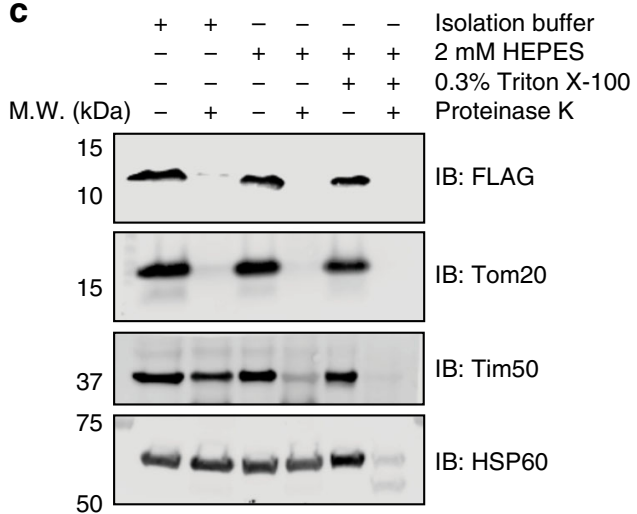

b

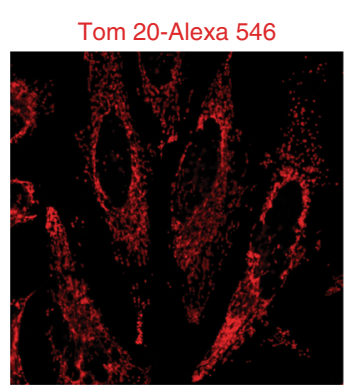

d

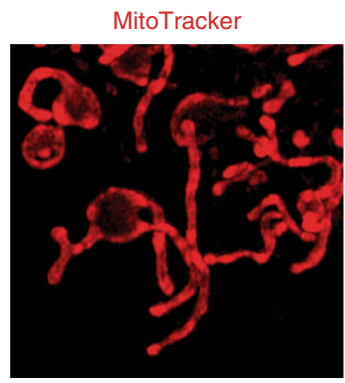

PIGBOS-FLAG-Alexa 488

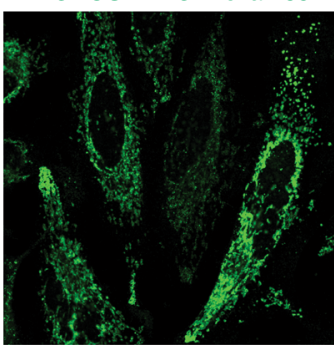

PIGBOS split GFP

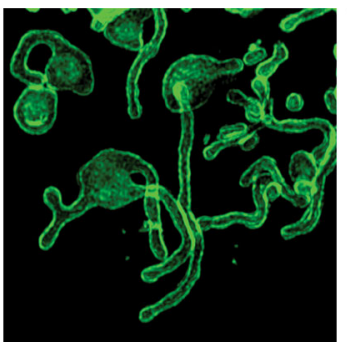

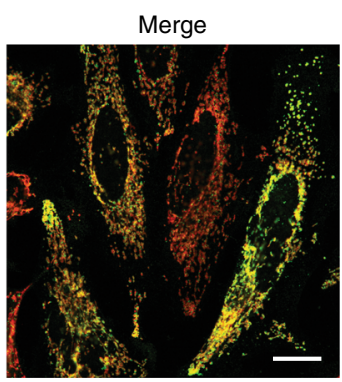

Merge

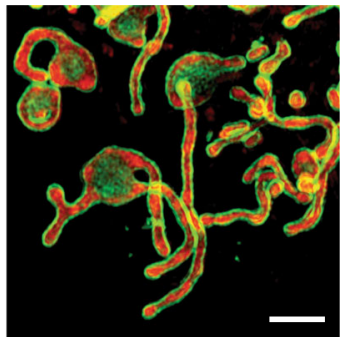

Fig. 2 PIGBOS is localized on the mitochondrial outer membrane (MOM). a Western blot analysis of nuclear, cytosolic, and mitochondrial fractions from HEK293T cells identifies PIGBOS as a mitochondrial microprotein. b Immunofluorescence imaging of HeLa cells after transfection with PIGBOS-FLAG reveals colocalization of PIGBOS (green) with the mitochondrial marker Tom20 (red), validating PIGBOS's mitochondrial localization. Scale bar: $20 \mu$ m. c Sub-mitochondrial localization using a protease protection assay reveals PIGBOS to be a mitochondrial outer membrane microprotein since proteinase $K$ can access and degrade PIGBOS without any mitochondrial permeabilization. $\mathbf{d}$ Live cell imaging of COS-7 cells that were co-transfected with PIGBOS-3 $\times$ GFP11 and GFP (1-10) results in a green fluorescent ring around the mitochondria (MitoTracker Deep Red FM), consistent with PIGBOS localization to the MOM. Scale bar: $2.5 \mu \mathrm{m}$

PIGBOS- $\Delta$ C-3 $\times$ GFP11 showed no detectable GFP signal, supporting that the C-terminus of PIGBOS is necessary for its interaction with CLCC1 (Fig. 4c, d and Supplementary Fig. 7).

The PIGBOS-CLCC1 interaction is not a tether. Given that CLCC1 and PIGBOS are ER and mitochondria transmembrane proteins, respectively, as well as the observation that PIGBOS- $3 \times$ GFP11 and CLCC1-GFP(1-10) generated the GFP fluorescence right between ER and mitochondria (Fig. 4a), we considered that they interact at ER-mitochondria contact sites. This model requires CLCC1 to be present in the portion of the ER that contacts the mitochondria which is referred to as the mitochondria-associated ER membrane (MAM). The MAM is an important inter-organelle junction in the cell that mediates cellular calcium levels, lipid metabolism, mitochondrial dynamics, and apoptosis ${ }^{30,31}$. We isolated the MAM using standard fractionation protocols ${ }^{32,33}$ and detected CLCC1 by Western blot along with the calnexin, a known MAM protein (Supplementary
Fig. 8a), which, consistent with our assumption, indicated that CLCC1 is in the ER fraction with close proximity to mitochondria.

There are only a handful of reported protein-protein interactions between the ER and mitochondria in mammalian cells, and most of these interactions are implicated in ER-mitochondria tethering $26,33,34$. For example, a known ER-mitochondria tether is comprised of the vesicle-associated membrane protein-associated protein $\mathrm{B} / \mathrm{C}(\mathrm{VAPB})$ in the ER and protein tyrosine phosphataseinteracting protein 51 (PTPIP51) in the mitochondria. Overexpression of VAPB and PTPIP51 increases the number of ERmitochondria contact sites ${ }^{35}$. To test a role for PIGBOS and CLCC1 in ER-mitochondria tethering, we used transmission electron microscopy to visualize ER-mitochondria contacts in WT and PIGBOS-KO U2OS cells (Fig. 5 and Supplementary Fig. 9). We observed no changes to ER-mitochondria contact sites (Fig. 5a), and quantitation of the normalized ER-mitochondria contact coefficient (ERMICC) ${ }^{36}$ revealed no significant differences in ERmitochondria contacts in WT versus PIGBOS-KO cells (Fig. 5b). 
a

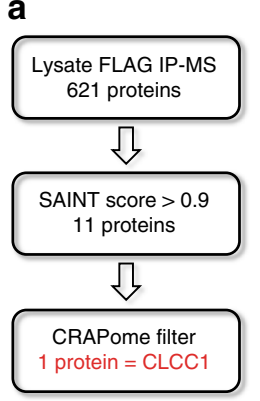

b

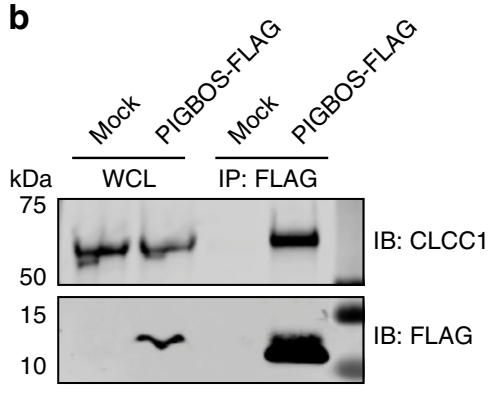

e

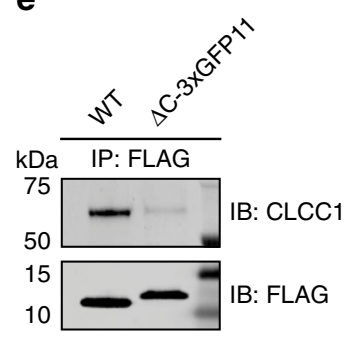

C

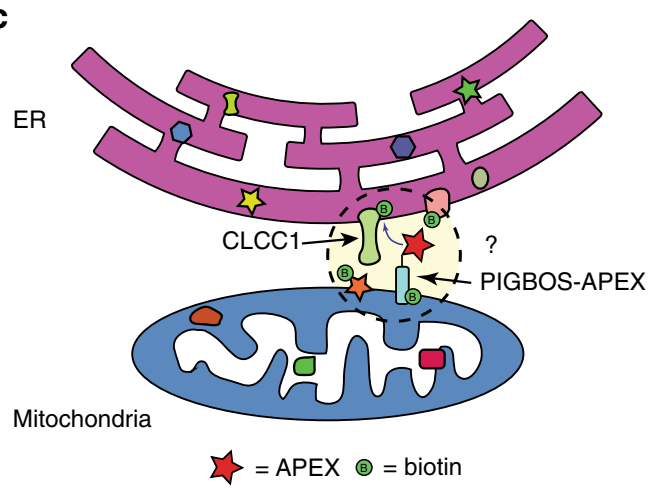

d

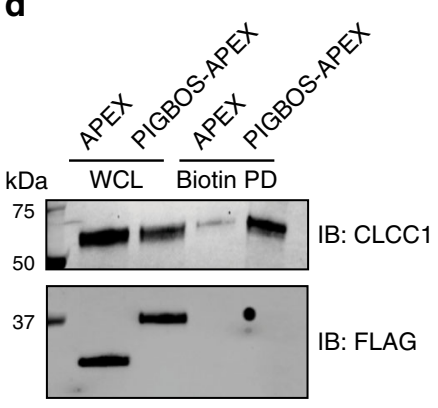

Fig. 3 PIGBOS interacts with the ER protein CLCC1. a Analysis of the PIGBOS-FLAG IP-MS to remove false positives and background proteins yielded CLCC1, an ER-resident protein, as a PIGBOS interacting protein. b Co-immunoprecipitation of PIGBOS-FLAG enriched CLCC1 from HEK293T total cell lysates. c A PIGBOS-APEX construct was used to proximity label the proteome and determine whether PIGBOS and CLCC1 are near each other in living cells. $\mathbf{d}$ Enrichment of CLCC1 in PIGBOS-APEX labeling proteome indicated that it was biotinylated and, therefore, in proximity to PIGBOS-APEX.

e Replacement of the PIGBOS C-terminal region with $3 \times$ GFP11 (PIGBOS- $\Delta$ C-3 $\times$ GFP11-FLAG) prevented enrichment of CLCC1

Consistent with the result that PIGBOS-CLCC1 is not a tether, overexpression of the known ER-mitochondria tether VAPB and PTPIP51 resulted in a change in ER-mitochondria morphology, in which the ER was localized near to mitochondria, whereas overexpression of PIGBOS and CLCC1 did not (Supplementary Fig. 10). In addition, the levels of CLCC1 in the MAM fraction from PIGBOS-KO and WT HEK293 cells are equivalent, which indicated that the amount of CLCC1 in the MAM does not depend on PIGBOS (Supplementary Figs. $8 \mathrm{~b}$ and 11).

Since the PIGBOS-CLCC1 interaction does not influence the ER-mitochondria contacts, we asked whether cells with changes to the number of ER-mitochondria contacts can regulate the interaction between PIGBOS and CLCC1. We tested this idea by measuring PIGBOS-CLCC1 split GFP intensity in cells expressing VAPB and PTPIP51. Using our quantitative flow cytometry assay, we found that the overexpression of VAPB and PTPIP51 significantly increased interactions between PIGBOS and CLCC1 as indicated by greater number of GFP positive cells and mean GFP intensity (Fig. 4e and Supplementary Fig. 12a), while total concentrations of PIGBOS and CLCC1 were unchanged (Supplementary Fig. 12b). This result demonstrated that the PIGBOS-CLCC1 interaction can be regulated by modulation of ER-mitochondria contacts, and further bolstered the model of CLCC1 and PIGBOS interacting at ERmitochondria contact sites.

PIGBOS modulates UPR in the ER. A genetics study in mice identified lower CLCC1 expression levels as the driver of a neurodegenerative phenotype, with mechanistic studies supporting increased UPR as the underlying cause ${ }^{27}$. We reproduced this finding and observed that treatment of CLCC1 knockdown (KD) cells with tunicamycin (TM), an inducer of ER stress, led to increased XBP1 splicing, an established marker for UPR (Supplementary Fig. 13a). Since PIGBOS interacts with CLCC1, we hypothesized that PIGBOS may have a role in UPR signaling, which, if true, would provide the first example of a mitochondrial protein regulator of UPR.

We tested whether PIGBOS regulates UPR by treating PIGBOS siRNA KD or CRISPR-Cas9 knockout (KO) cells (Supplementary Figs 11 and 13b) with TM. In the absence of PIGBOS, we observed an increased sensitivity of cells to TM-a stronger UPR at lower TM concentrations-detected as elevated levels of spliced XBP1 and an increased ratio of spliced XBP1 to unspliced XBP1 (XBP1s/XBP1u) (Fig. 6a and Supplementary Fig. 13c-e). Furthermore, expression of an siRNA resistant PIGBOS-FLAG construct in the PIGBOS-KD cells partially reversed the phenotype by normalizing the sensitivity of UPR to TM (Fig. 6a), verifying that PIGBOS is responsible for the observed effects on UPR. Overexpression of PIGBOS in WT cells resulted in the desensitization of cells to UPR with decreased XBP1 splicing (Supplementary Fig. 13f), providing additional evidence for a specific role for PIGBOS in UPR. Taken together, these results suggested that modulating PIGBOS levels can in turn modulate cellular sensitivity towards ER stress.

To assess the generality of PIGBOS regulation of UPR, we tested different ER stressors and measured the activity of the ATF6 branch of the UPR pathway. Loss of PIGBOS also showed heightened sensitivity to thapsigargin (TG) and Brefeldin A (BFA), two mechanistically distinct UPR activators, indicating that PIGBOS is downstream of both types of UPR induction (Fig. 6b and Supplementary Fig. 13g). Analysis of the ATF6 branch of UPR in WT and PIGBOS-KD cells using an ATF6 luciferase reporter assay ${ }^{37}$ revealed increased ATF6-driven luciferase activity in PIGBOS-KD cells, which was rescued by the expression of siRNA resistant PIGBOS-FLAG (Fig. 6c). To 
a
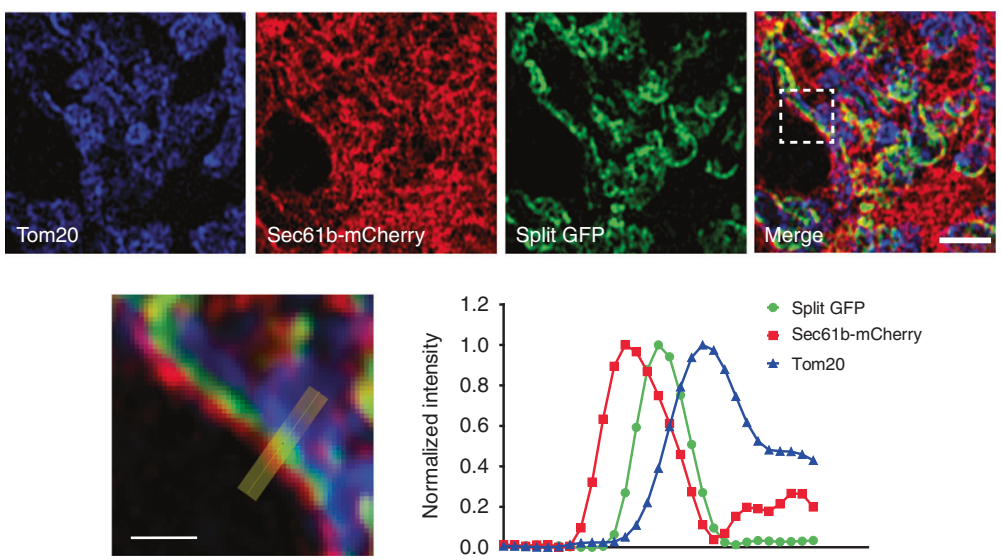

b
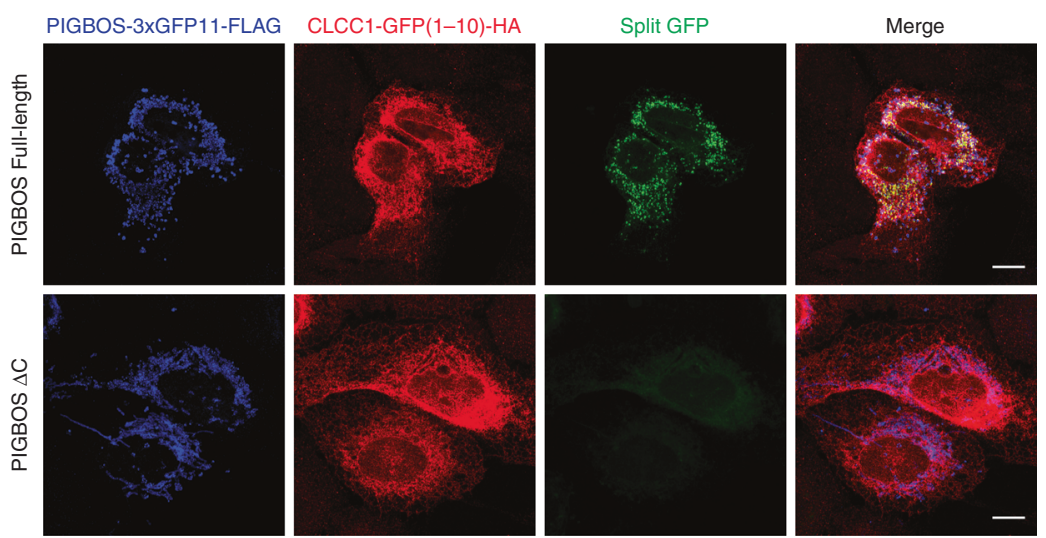

C
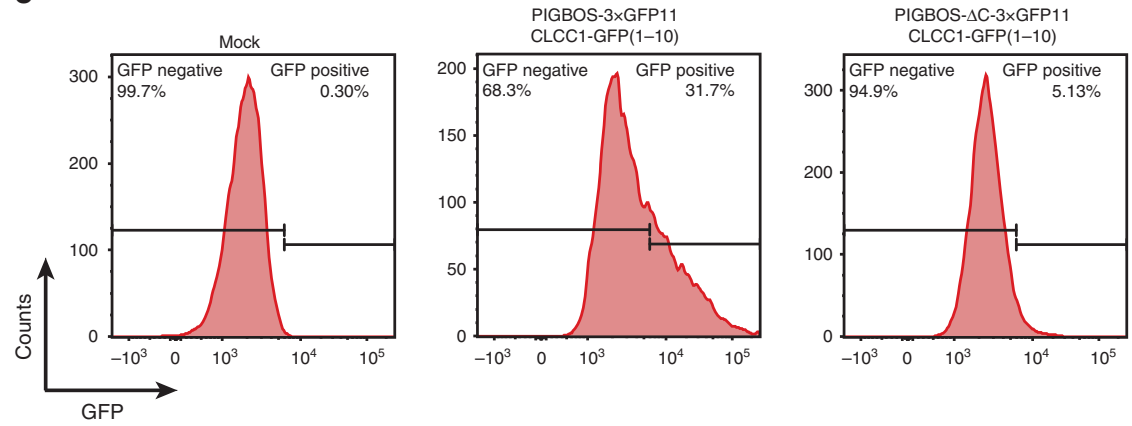

d
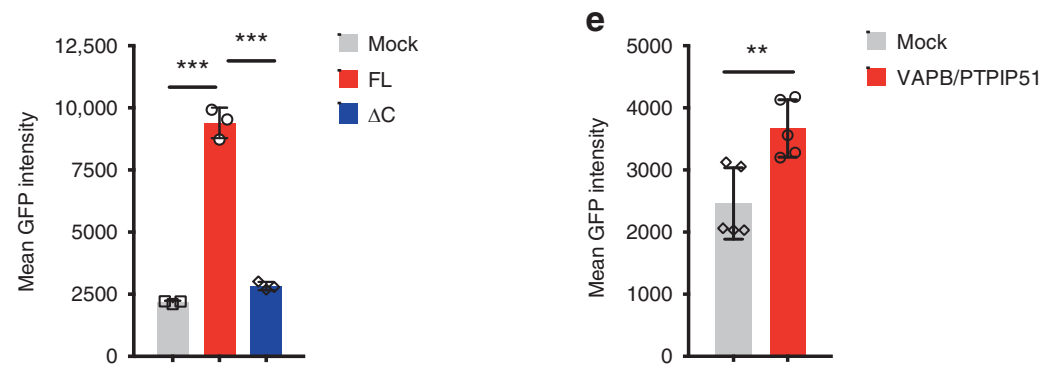

obtain the most comprehensive view of PIGBOS in its regulation of UPR, we measured mRNA levels of a panel of UPR-regulated genes that promote degradation of misfolded proteins (ERdj4 and EDEM1), protein folding (HYOU1, GRP78, and ERO1LB), and apoptosis $(C H O P)$. Upon UPR induction with TM, the loss of PIGBOS led to dramatic increases in the levels of all UPR target genes measured, indicating increased UPR signaling across all the branches (IRE1, PERK, and ATF6) (Fig. 6d and Supplementary Fig. 14a). Meanwhile, PIGBOS overexpressing cells showed the opposite effect, in which the UPR target genes showed less UPR activation, indicating a tunable modulation of ER stress by PIGBOS microprotein levels (Supplementary Fig. 14b). We then confirmed via Western blot that TM treatment of PIGBOS-KD cells led to higher ATF4 and CHOP protein levels (Supplementary Fig. 13h). These data identified PIGBOS as a heretofore unknown mitochondrial regulator of UPR, and the only known microprotein linked to the regulation of cell stress or interorganelle signaling. 
Fig. 4 Validation of PIGBOS-CLCC1 interaction via split GFP bimolecular complementation. a (Top) Transfection of COS-7 cells with PIGBOS-3 $\times$ GFP11 and CLCC1-GFP(1-10) resulted in a GFP signal, which could only occur if the two proteins are close enough to interact and reconstitute a functional GFP. Scale bar: $2 \mu \mathrm{m}$. (Bottom) The region in the white box was enlarged, and a cross-sectional analysis of the normalized fluorescence distribution of the Tom20 (MOM), Sec61b (ER), and GFP signals places the GFP signal between the ER and MOM. Scale bar: $0.5 \mu \mathrm{m}$. b U2OS cells were co-transfected with CLCC1GFP(1-10)-HA and PIGBOS-3 $\times$ GFP11-FLAG (or PIGBOS- $\triangle$ C-3 $\times$ GFP11-FLAG). Forty-eight hours later, cells were fixed and stained with FLAG and HA antibodies overnight before imaging. Scale bar: $10 \mu \mathrm{m}$. c Flow cytometry measurement of PIGBOS-CLCC1 interaction in HEK293T cells. HEK293T cells were co-transfected with CLCC1-GFP(1-10)-HA and PIGBOS-3 $\times$ GFP11-FLAG (or PIGBOS- $\Delta$ C-3 $\times$ GFP11-FLAG). GFP signals were assessed by flow cytometry 72 hours after transfection. d Quantification of mean GFP intensity in (c). Error bars, s.d., ${ }^{\star \star \star} p<0.001$ (two tailed unpaired $t$-test), $n=3$ independent experiments. e Flow cytometry measurement of reconstituted PIGBOS-CLCC1 split GFP intensity in HEK293T cells expressing a known ER-mitochondrial tether, VAPB/PTPIP51. Error bars, s.d., ${ }^{\star \star} p<0.01$ (two tailed unpaired $t$-test), $n=5$ independent experiments. Source data for Fig. $4 a$, $d$ and e are provided as a Source Data file

a

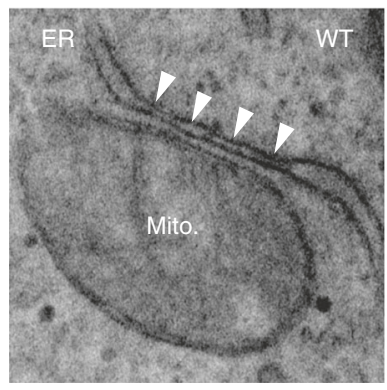

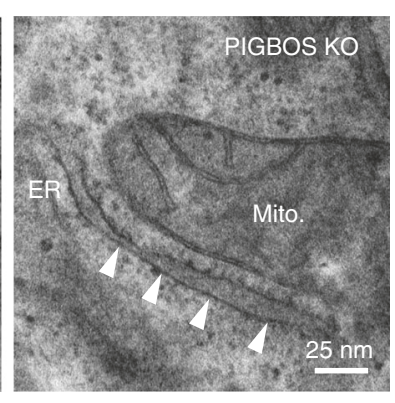

b

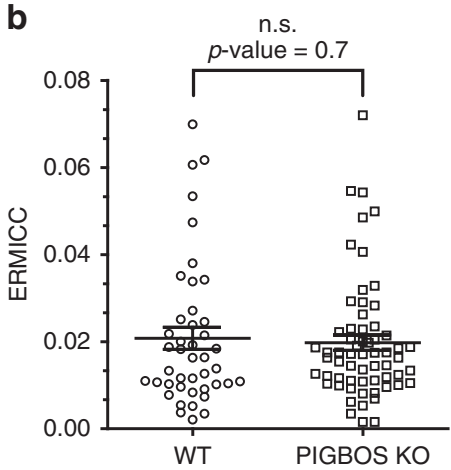

Fig. 5 PIGBOS shows no effect on modulation of ER-mitochondria contacts. a Representative transmission electron microscopy images from WT and PIGBOS-KO U2OS cells showed no remarkable differences in ER-mitochondria contacts. ER and mitochondria contact sites are indicated by white arrows. b Quantitation of the normalized ER-mitochondria contact coefficient (ERMICC) did not identify a significant difference between ERMICC of WT vs. PIGBOS-KO U2OS cells. Data are collected from two independent experiments and pooled from 43 WT mitochondria (eight cells), and 62 PIGBOS-KO mitochondria (seven cells) and the bar graph is the ERMICC \pm s.e.m. with the $p$-value calculated using two tailed unpaired $t$-test with all data points included in the calculation. Source data for Fig. $5 b$ are provided as a Source Data file

We then assessed whether the PIGBOS-CLCC1 interaction is regulated by ER stress. Immunoprecipitation of PIGBOS-FLAG in TM or TG treated cells showed similar amounts of co-eluted CLCC1, suggesting ER stress has no effect on the PIGBOSCLCC1 interaction (Supplementary Fig. 15a). To confirm this result, we used flow cytometry analysis to evaluate PIGBOSCLCC1 split GFP intensity in TM or TG treated cells. No significant differences with regard to either GFP positive cells or mean GFP intensity were observed in cells with ER stress up to $7 \mathrm{~h}$ (Supplementary Fig. 15b-d).

UPR is a dedicated signaling network to deal with unfolded protein stress in the ER. Recent studies revealed emerging evidence of a cellular response to unfolded protein accumulation in the mitochondria, referred to as MitoUPR, which is induced by distinct stimuli and results in different response mechanisms with some overlapping targeting genes compared to the canonical ER $\mathrm{UPR}^{38,39}$. To test whether PIGBOS has a role in MitoUPR, we used bardoxolone (CDDO) to chemically induce mitochondrial protein misfolding in PIGBOS-KO and WT cells, and evaluated the MitoUPR targeting gene expression (i.e., $H S P D 1$ and $C H O P)^{40,41}$. Both genes increased dramatically upon CDDO treatment, however, there was no notable difference between PIGBOS-KO and WT cells (Supplementary Fig. 16). It is worth noting that CHOP level was significantly increased in PIGBOS-KD cells compared to WT cells in response to TM induced ER stress, indicating that PIGBOS specifically regulates ER UPR.

PIGBOS regulates ER stress-induced apoptosis. Previous studies demonstrated that prolonged ER stress would lead to apoptosis if cells fail to cope with accumulating misfolded proteins ${ }^{9}$. Since cells lacking PIGBOS are more sensitive to UPR, we predicted that these cells would undergo apoptosis more readily. Indeed, we observed increased apoptosis in PIGBOS-KD and PIGBOS-KO cells treated with TG or TM using a caspase- 3 and PARP-cleavage assay (Fig. 6e, f, and Supplementary Fig. 17a-c). PIGBOS-KD cells were also less viable than control cells during TG-induced cell stress (Fig. $6 \mathrm{~g}$ ). Interestingly, treating cells with staurosporine (STS), a non-selective protein kinase inhibitor and apoptosis inducer, revealed neglectable differences of cell viability in PIGBOS-KD and WT cells, which indicated a specific connection between ER stress and PIGBOS regulation (Supplementary Fig. 17d). Together, these results showed that loss of PIGBOS increases cellular sensitivity to ER stress, which in turn increases apoptosis and links PIGBOS levels to the ability of cells to survive stress.

PIGBOS-CLCC1 interaction is necessary for PIGBOS function. To confirm that the increased UPR sensitivity in PIGBOS diminished cells is mediated by PIGBOS-CLCC1 interaction, we performed rescue experiments with non-CLCC1 binding PIGBOS mutants. Only full-length PIGBOS microprotein reversed the XBP1 splicing phenotype, whereas the non-CLCC1-binding PIGBOS mutants with the C-terminus truncation (PIGBOS- $\Delta \mathrm{C}$ $3 \times$ GFP11-FLAG) or the triple alanine mutant of aa $30-32$ (PIGBOS-3 $\times$ GFP11-FLAG, 30-32 AAA) showed similar activity as in cells lacking PIGBOS (Fig. 6h and Supplementary Fig. 18a). Furthermore, expression of PIGBOS full-length protein, but not C-terminus truncated PIGBOS (PIGBOS- $\Delta$ C- $3 \times$ GFP11-FLAG), in PIGBOS-KD cells is able to partially rescue the ER stress triggered apoptosis (Supplementary Fig. 18b). In addition, we made chimeric PIGBOS variants to anchor the PIGBOS cytosolic region (i.e., aa 30-54) to either the ER membrane (C1-PIGBOS 
a

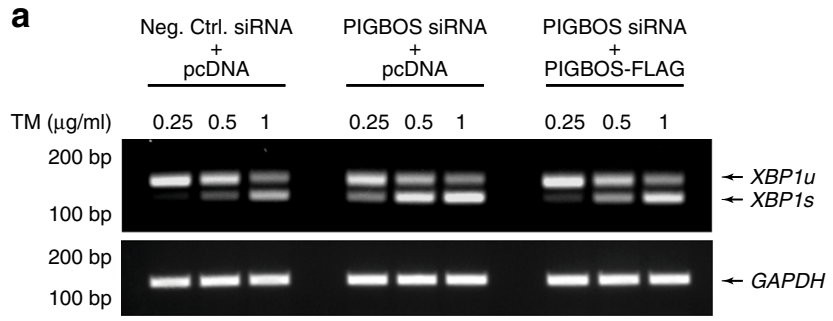

b

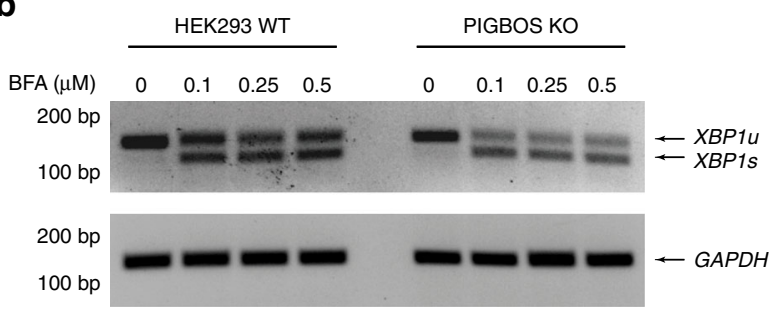

e
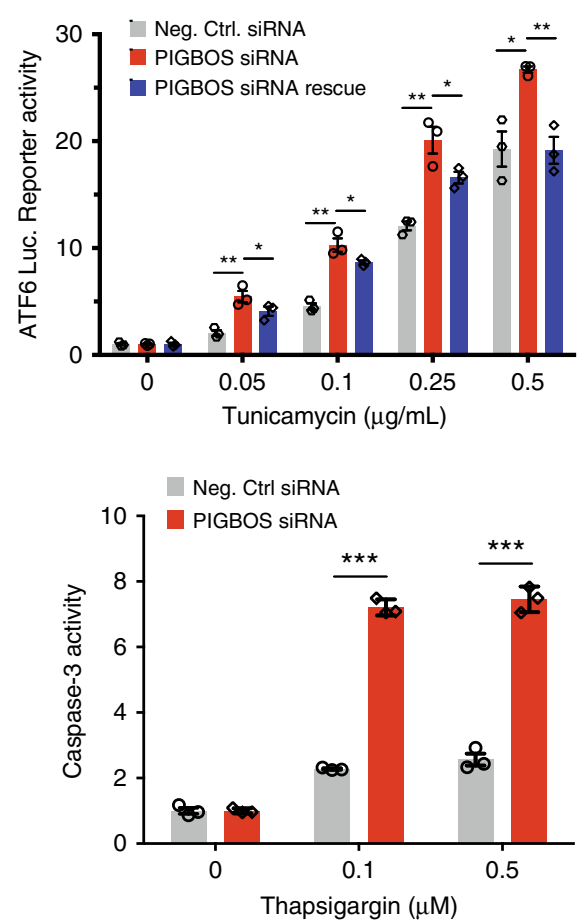

g

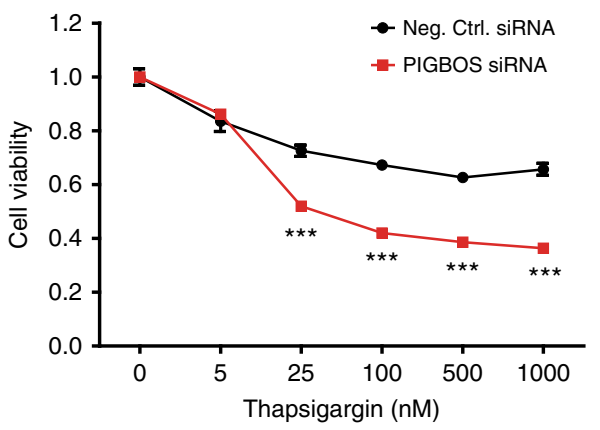

d

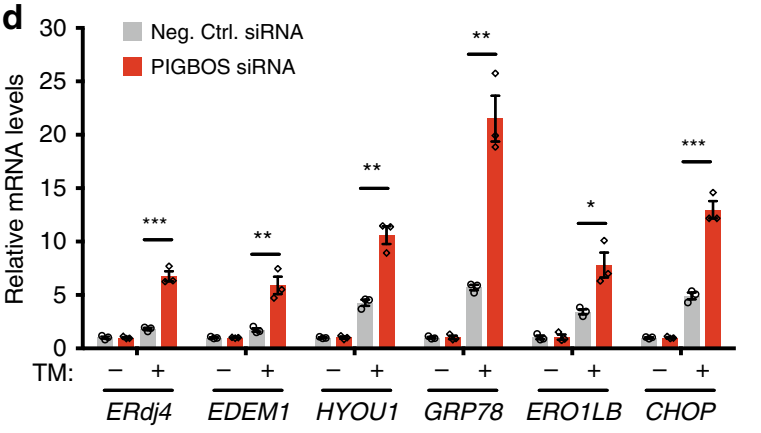

$\mathbf{f}$

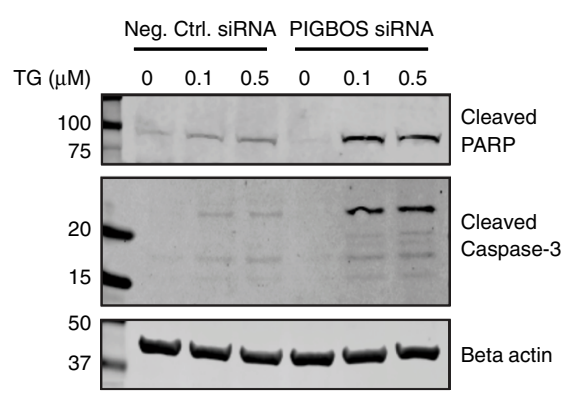

h

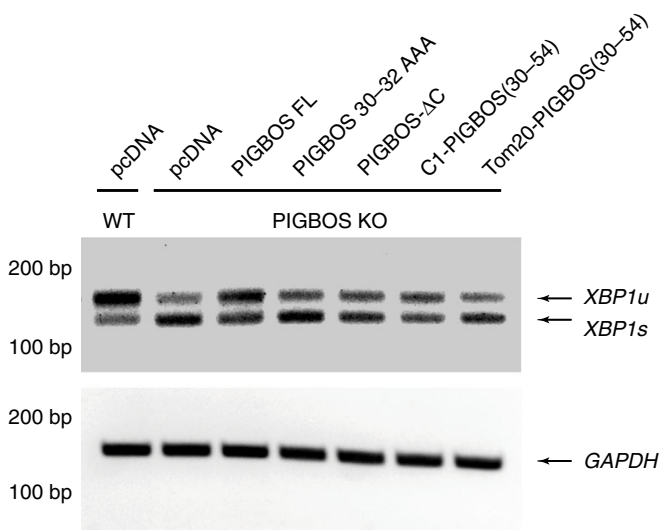

Fig. 6 PIGBOS regulates the amplitude of UPR, and apoptosis. a PIGBOS-KD and control HEK293 cells were treated with indicated concentrations of tunicamycin (TM) followed by RT-PCR analysis of XBP-1 splicing (unspliced XBP1 (XBP1u) and spliced XBP1 (XBP1s)). GAPDH was used as a loading control. A stronger UPR correlate with higher XBP1s/XBP1u ratio in PIGBOS-KD cells, which could be rescued by expression of a siRNA-resistant PIGBOSFLAG. b XBP-1 mRNA splicing was measured in PIGBOS-KO and control HEK293 cells treated with indicated doses of brefeldin A (BFA) for $3 \mathrm{~h}$. c ATF6dependent luciferase reporter measures the activation of another branch of the UPR pathway. PIGBOS-KD led to increased luciferase activity indicative of a greater UPR, and the expression of the siRNA-resistant PIGBOS-FLAG reversed this effect. $\mathbf{d}$ RT-qPCR quantitation of a panel of UPR target genes in PIGBOS-KD and control HEK293 cells after an 8-hour treatment with vehicle or $1 \mu \mathrm{g} / \mathrm{ml}$ of TM. e Caspase-3 activity in mock and PIGBOS-KD U2OS cells treated with thapsigargin (TG) for $27 \mathrm{~h}$. f Cleaved PARP and caspase-3 levels were measured by Western blot in PIGBOS-KD and control U2OS cells treated with TG for $27 \mathrm{~h} . \mathbf{g}$ PIGBOS-KD and control U2OS cells were treated with indicated doses of TG for $48 \mathrm{~h}$ followed by cell viability measurements using MTT. h HEK293 PIGBOS-KO and WT cells were transfected with PIGBOS variants constructs as indicated. Forty-eight hours later, cells were incubated with $1 \mu \mathrm{g} / \mathrm{ml}$ of tunicamycin for $3 \mathrm{~h}$. XBP1 splicing activity was measured by RT-PCR. Error bars, s.e.m. The $p$-values were calculated using two tailed unpaired $t$-test. ${ }^{\star} p<0.05,{ }^{\star \star} p<0.01,{ }^{\star \star \star} p<0.001, n=3$ experiments. Source data for Fig. $6 c-e$ and $6 \mathrm{~g}$ are provided as a Source Data file 
(30-54)) or MOM (Tom20-PIGBOS(30-54)). Confocal images demonstrated accurate subcellular localization (Supplementary Fig. $18 \mathrm{c}$ and d), but they were unable to interact with CLCC1 efficiently (Supplementary Fig. 18e). In addition, Tom20-PIGBOS (30-54) failed to rescue XBP1 splicing activity, while C1-PIGBOS (30-54) showed decreased XBP1 splicing activity but this might be due to $\mathrm{C} 1$ peptide having independent activity at the $\mathrm{ER}^{42}$ (Fig. 6h and Supplementary Fig. 18a).

The Tom20-PIGBOS(30-54) experiment demonstrates that placing the PIGBOS C-terminal region at MOM is insufficient for mediating the PIGBOS-CLCC1 interaction and regulating the ER stress response. As a small protein, we suspect that the different parts of PIGBOS are not easily separated from each other into functional domains, which is why we refer to them as regions. Unlike large, multidomain proteins the separation and swapping of the C-terminal region may affect the secondary or tertiary structure of PIGBOS, and in the process inhibit its ability to bind CLCC1 or partake in UPR signaling. PIGBOS belongs to a class of single-pass transmembrane proteins in which the transmembrane regions (oligomerization state, tilt angle and topology) are essential to control structure and function. TMDOCK analysis predicts that the PIGBOS transmembrane region is a homodimer (Supplementary Fig. 19a), providing evidence for PIGBOS having a tertiary structure ${ }^{43}$. To test this prediction, we transfected cells with human PIGBOS-FLAG and human PIGBOS-HA, or human PIGBOS-FLAG and rat PIGBOS-HA and performed FLAG immunoprecipitations. We found human PIGBOS-FLAG interacts with human and rat PIGBOS-HA providing experimental evidence for a PIGBOS oligomerization and a tertiary structure for this microprotein (Supplementary Fig. 19b, c). PIGBOS might make up for its diminutive size by oligomerizing into a tertiary structure that can provide a highly specific and unique protein structure necessary for CLCC1 binding. Future structure studies of PIGBOS and PIGBOS-CLCC1 complex will elucidate this structure in greater detail and define the role of PIGBOS tertiary structure in PIGBOS function.

\section{Discussion}

Understanding how cells respond to stress is of importance. These pathways are required for maintaining homeostasis and cell health, and their dysregulation can lead to disease. For example, UPR dysfunction contributes to accumulation of key disease-related proteins, and thus plays an essential role in the pathogenesis of many neurodegenerative disorders, including Alzheimer's disease, Parkinson's disease, and Huntington's disease ${ }^{14,15}$. Cells with an insufficient capacity to handle protein production begin to accumulate unfolded or misfolded proteins, which causes ER stress and triggers UPR. Elegant genetic studies in yeast revealed the conserved ER machinery that is activated during UPR and mediates the signaling pathways needed to express the genes necessary to cope with stress. The essential proteins in the eukaryotic ER stress response machinery include the kinases PERK and IRE1, and the proteolytically activated transcription factor ATF6. All three of these foundational genes are localized to the ER, identifying the ER as the hub for regulating UPR.

The ER stress response consists of intricate signaling networks across the entire cell with spatial and temporal regulation that requires communications between ER and other intracellular organelles. In particular, mitochondria play a vital role in crosstalk with ER during UPR by providing energy for protein folding in the ER as well as activating apoptosis if the stress remains unmitigated. However, the communication between the ER and mitochondria in the context of UPR is elusive. One way to solve how ER-originating cellular signaling pathways such as UPR are propagated in the cell is to identify regulators of inter-organelle communication at contact sites. The list of known ERmitochondria contact proteins in mammals is relatively short ${ }^{34}$, and only one of these proteins, the mitochondrial fusion protein Mfn2, has been implicated in $\mathrm{UPR}^{44}$. The role of Mfn2 is confounding because it is both an ER and mitochondrial resident protein and it is a tether that regulates ER-mitochondria contact sites $^{36,45}$, which makes it difficult to dissect whether Mfn2 directly controls UPR or whether another protein complex at ERmitochondria contact sites might be responsible and is coincidentally disrupted by the loss of Mfn2. The microprotein PIGBOS is the latest member to join the short list of functional protein complexes at the ER-mitochondria interface but is the only mitochondria-specific protein to date to modulate UPR in the ER.

PIGBOS has a unique genomic localization, which is on the opposite strand of the PIGB gene. In human, the first exons of these two genes share an overlapping region, raising the question as to whether expression of one gene can influence the other, or whether the two genes are co-regulated. We knocked down PIGBOS and PIGB respectively in HEK293 cells using siRNAs, and measured gene expression levels of the other gene. We found that $\mathrm{KD}$ of one gene does not interfere with the expression of the other one (Supplementary Fig. 20a, b). In addition, we demonstrated that PIGBOS mRNA levels decreased in LPS treated RAW 264.7 cells. Interestingly, PIGB mRNA also decreased to a similar extent in the same cells (Supplementary Fig. 20c). These results implied that PIGBOS and PIGB might be co-regulated by the same promoter and transcription factors. Future work will be performed to fully address this question and investigate whether it can be applied to other antisense microproteins.

The characterization of microproteins has led to unprecedented mechanistic insights in other pathways, such as the role of minion in muscle fiber formation ${ }^{7}$ and CYREN in the cell-cycle dependent inhibition of non-homologous end joining (NHEJ) repair $^{8}$. Functional microproteins, including PIGBOS, demonstrate that they are as vital to cellular and physiological functions as any other protein; however, unlike large multidomain proteins, microproteins present challenges in trying to understand their structure-function relationship. For example, due to the small size, normal protein immunoprecipitation experiments might not be able to enrich the binding partners because microproteins may not have enough surface area to bind an antibody while maintaining a microprotein-protein interaction partner ${ }^{46}$. As a result, immunoprecipitation with overexpressed epitope-tagged microproteins is the most reasonable way to characterize the interactomes of microproteins and has successfully been used to find several bona fide microprotein-protein interactions $s^{1,8,23}$. Furthermore, microprotein regions, as opposed to classical protein domains, cannot be swapped and retain their functions as demonstrated by TOM20-PIGBOS(35-54) fusion which loses PIGBOS's binding and biological activity.

The functional assignment of PIGBOS in this study has revealed the ability of a mitochondrial protein to regulate UPR in the ER, which makes PIGBOS unique and demonstrates the existence of non-ER proteins in the regulation of UPR. Our work also reveals that inter-organelle interactions can be mediated by microproteins and raises the possibility that other inter-organelle or inter-cellular protein interactions at membrane contact sites might involve microproteins. Furthermore, recent work has demonstrated that the dysregulation of the ER stress response is implicated in human disease including viral infections, neurodegeneration, cancer, and diabetes ${ }^{47}$. Given the importance of UPR in biology and disease, future studies on PIGBOS's role in UPR should afford additional insights and may provide methods for regulating this pathway for therapeutic applications. 


\section{Materials and methods}

Materials. Cell lines used in the study were purchased from ATCC, HEK293 (CRL-1573), HEK293T (CRL-11268), U2OS (HTB-96), HeLa (CCL-2), and COS-7 (CRL-1651). siRNAs used in this study were purchased from GE Healthcare Dharmacon, Inc. and listed in Supplementary Table 3. Sequences of RT-qPCR primers are listed in Supplementary Table 4. Sequences of gRNA protospacers and genotyping primers for PIGBOS knockout are shown in Supplementary Table 5. Information of antibodies used in this study are shown in Supplementary Table 6. DNA constructs and corresponding subcloning primers are listed in Supplementary Tables 7 and 8 respectively.

Animal care. All animal procedures were approved by the Institutional Animal Care and Use Committee of the Salk Institute and were conducted in accordance with the PHS Policy on Humane Care and Use of Laboratory Animals (PHS Policy, 2015), the U.S. Government Principles for Utilization and Care of Vertebrate Animals Used in Testing, Research and Training, the NRC Guide for Care and Use of Laboratory Animals (8th edition) and the USDA Animal Welfare Act and Regulations. All animals were housed in an AAALAC accredited facility in a climate-controlled environment (65-72 degrees Fahrenheit, 30-70\% humidity) under 12-h light/12-h dark cycles. Upon arrival, animals were physically examined by veterinary staff for good health and acclimated for at least two weeks prior to initiation of antiserum production. Each animal was monitored daily by the veterinary staff for signs of complications and weighed every two weeks. Routine physical exams were also performed by the veterinarian quarterly on all rabbits and guinea pigs. For the production of antiserum against human PIGBOS, three 10 to 12 -week old, female New Zealand white rabbits, weighing $3.0-3.2 \mathrm{~kg}$ at beginning of the study, were procured from Irish Farms (I.F.P.S. Inc., Norco, California, USA). Rabbits were provided with ad libitum feed (5326 Lab Diet High Fiber), micro-filtered water and weekly fruits, vegetables and alfalfa hay for enrichment For the production of rat PIGBOS antiserum in guinea pigs, four 10-12-week old, female Hartley guinea pigs, weighing 700-750 g at the beginning of the study, were procured from Charles River Laboratories. Guinea pigs were provided with ad libitum feed (5025 Lab Diet), micro-filtered water and weekly fruits and vegetables for enrichment.

Preparation of antigens. Peptides were synthesized by RS Synthesis (Louisville, KY), HPLC purified to $>95 \%$, and amino acid sequence verified by mass spectrometry. Peptides were conjugated to maleimide activated Keyhole Limpet Hemocyanin (KLH) per manufacturer's instructions (ThermoFisher, Waltham MA). Specific peptides used to generate antisera were as follows: Cys32 human PIGBOS(32-42)- $\mathrm{NH}_{2}$, CAKDQKELKEK- $\mathrm{NH}_{2}$; Cys32 rat PIGBOS (32-54), CSRDQKELKELVKILQESEEKRS.

Injection and bleeding of animals. The antigen was delivered to host animals using multiple intradermal injections of the peptide-KLH conjugate in Complete Freund's Adjuvant (initial inoculation) or incomplete Freund's adjuvant (booster inoculations) every three weeks for rabbits and once every four weeks for guinea pigs. Animals were bled, $<10 \%$ total blood volume, one week (rabbits) or two weeks (guinea pigs) following booster injections and bleeds screened for titer and specificity. Rabbits were administered 1-2 mg/kg Acepromazine IM prior to injections of antigen or blood withdrawal. Guinea pigs were anesthetized using inhalation isoflurane maintained at $2-2.5 \%$ prior to injections and bleedings. At the termination of the study, rabbits were exsanguinated under anesthesia (ketamine $50 \mathrm{mg} / \mathrm{kg}$ and acepromazine $1 \mathrm{mg} / \mathrm{kg}$, IM) and euthanized with an overdose of pentobarbital sodium and phenytoin sodium $(1 \mathrm{ml} / 4.5 \mathrm{~kg}$ of body weight IC to effect). Guinea pigs were exsanguinated via cardiac puncture under inhalation anesthesia (isoflurane maintained at 2-2.5\%). After blood was collected death of animals was confirmed. All animal procedures were conducted by experienced veterinary technicians, under the supervision of Salk Institute veterinarians.

Characterization and purification of antisera. Each bleed from each animal was tested at multiple doses for the ability to recognize the synthetic peptide antigen; bleeds with highest titers were further analyzed by western immunoblot for the ability to recognize the full-length endogenous protein and to check for crossreactivity to other proteins. Antisera with the best characteristics of titer against the synthetic peptide antigen, ability to recognize the endogenous protein, and specificity were antigen affinity purified and used for all studies. Rabbit PBL\#7410 antihuman PIGBOS and guinea pig PBL\#114 anti-rat PIGBOS were purified using human PIGBOS(32-54) coupled to Affi-Gel 10 (Bio-Rad Laboratories, Hercules CA) or Cys32 rat PIGBOS (32-54) covalently attached to Sulfolink agarose (ThermoFisher, Waltham MA), respectively. Coupling of peptides to resins was per manufacturer's instructions. To ensure that the same batch of purified antibodies could be used for this and future studies, large volumes, $\sim 20 \mathrm{ml}$ sera, from bleeds with similar profiles were purified.

MS sample preparation and instrumentation. Samples were precipitated with trichloroacetic acid (TCA, MP Biomedicals, \#196057) overnight at $4{ }^{\circ} \mathrm{C}$. Dried pellets were dissolved in $8 \mathrm{M}$ urea, reduced with $5 \mathrm{mM}$ tris (2-carboxyethyl) phosphine hydrochloride (TCEP, Thermo, \#20491) and alkylated with $10 \mathrm{mM}$ iodoacetamide (Sigma, I1149). Proteins were then digested overnight at $37^{\circ} \mathrm{C}$ with trypsin (Promega, V5111). The reaction was quenched with formic acid at a final concentration of $5 \%(\mathrm{v} / \mathrm{v})$. The digested samples were analyzed on a $\mathrm{Q}$ Exactive mass spectrometer (Thermo). The digest was injected directly onto a $30 \mathrm{~cm}, 75 \mu \mathrm{m}$ ID column packed with BEH $1.7 \mu \mathrm{m}$ C18 resin (Waters). Samples were separated at a flow rate of $200 \mathrm{nl} / \mathrm{min}$ on a nLC 1000 (Thermo). Buffer A and B were $0.1 \%$ formic acid in water and acetonitrile, respectively. A gradient of 5-40\% B over $110 \mathrm{~min}$, an increase to $50 \% \mathrm{~B}$ over $10 \mathrm{~min}$, an increase to $90 \% \mathrm{~B}$ over another $10 \mathrm{~min}$ and held at $90 \% \mathrm{~B}$ for a final $10 \mathrm{~min}$ of washing was used for $140 \mathrm{~min}$ total run time. The column was re-equilibrated with $20 \mu \mathrm{l}$ of buffer A prior to the injection of sample. Peptides were eluted directly from the tip of the column and nanosprayed directly into the mass spectrometer by application of $2.5 \mathrm{kV}$ voltage at the back of the column. The Q Exactive was operated in a data-dependent mode. Full MS1 scans were collected in the Orbitrap at $70 \mathrm{~K}$ resolution with a mass range of $400-1800 \mathrm{~m} / \mathrm{z}$ and an AGC target of $5 \mathrm{e}^{6}$. The ten most abundant ions per scan were selected for MS/MS analysis with HCD fragmentation of 25NCE, an AGC target of $5 \mathrm{e}^{6}$ and minimum intensity of $4 \mathrm{e}^{3}$. Maximum fill times were set to $60 \mathrm{~ms}$ and $120 \mathrm{~ms}$ for MS and MS/MS scans respectively. Quadrupole isolation of $2.0 \mathrm{~m} / \mathrm{z}$ was used, dynamic exclusion was set to $15 \mathrm{~s}$ and unassigned charge states were excluded. Protein and peptide identification were done with Integrated Proteomics Pipeline-IP2 (Integrated Proteomics Applications). Tandem mass spectra were extracted from raw files using RawConverter ${ }^{48}$ and searched with ProLuCID ${ }^{49}$ against human UniProt database appended with microprotein sequences. The search space included all fully-tryptic and half-tryptic peptide candidates with a maximum of two missed cleavages. Carbamidomethylation of cysteine was counted as a static modification. Data was searched with $50 \mathrm{ppm}$ precursor ion tolerance and $50 \mathrm{ppm}$ fragment ion tolerance. Data was filtered to $10 \mathrm{ppm}$ precursor ion tolerance post search. Identified proteins were filtered using DTASelect ${ }^{50}$ and utilizing a target-decoy database search strategy to control the false discovery rate to $1 \%$ at the protein level.

Extraction of rat tissues for Western blot analysis. Tissues were extracted using a mixture of hot $\left(90^{\circ} \mathrm{C}\right) 1 \mathrm{~N}$ acetic acid/ $0.1 \mathrm{~N} \mathrm{HCl}$, homogenized with a Polytron blender, centrifuged at $30,000 \times g$ for $30 \mathrm{~min}$ at $4{ }^{\circ} \mathrm{C}$, and supernatants removed and filtered through $5 \mu \mathrm{m}$ syringe filters. Supernatants were enriched for microproteins as described ${ }^{51}$, except that Bond Elut C18 cartridges were used.

Confocal imaging. For fixed cell imaging, cells were seeded onto coverslips (Fisher Scientific, 12-541-B) pre-treated with $50 \mu \mathrm{g} / \mathrm{mL}$ poly-L-lysine (Sigma, P1399). The next day, cells were transfected with constructs as indicated using Lipofectamine 2000. Tweny-four or forty-eight hours post-transfection, cells were fixed with $4 \%$ paraformaldehyde (Polysciences, Inc., \#18814) and permeabilized with fresh $0.1 \%$ saponin (Alfa Aesar, A18820). After incubating with 4\% BSA in PBS for 1 hour at room temperature, cells were stained with corresponding primary antibodies overnight at $4{ }^{\circ} \mathrm{C}$. Then the cells were washed three times with PBS, followed by incubating with Alexa Fluor-labeled secondary antibodies for 1 hour at room temperature. If necessary, nuclei were counterstained with Hoechst 33258 (Sigma, \#94403, 1:2000 in PBS). After three PBS washes, the coverslip was mounted on slides using Prolong ${ }^{\oplus}$ Gold Antifade Mountant (Life Technologies, P36930). For live cell imaging, COS-7 cells were seeded onto 4 -well chambered cover glass (Cellvis, C4-1.5H-N), which was pre-treated with $50 \mu \mathrm{g} / \mathrm{mL}$ poly-L-lysine (Sigma $\mathrm{P} 1399)$. The next day, cells were transfected with constructs as indicated using Lipofectamine 2000. Twenty-four hours post-transfection, cells were treated with MitoTracker Deep Red FM (Life Technologies, M22426) to label mitochondria. Cell culture medium was then changed to phenol-red free DMEM $+10 \%$ FBS and imaged at $37^{\circ} \mathrm{C}$ and $5 \% \mathrm{CO}_{2}$. All samples were imaged using a Zeiss LSM 880 Airyscan confocal microscope with a $63 \times 1.4 \mathrm{NA}$ oil immersion objective at $2 \times$ Nyquist pixel and $\mathrm{z}$-stack step sizes, then processed using automatic filter settings in Zen Black (Zeiss) software. Images were then analyzed using FIJI software.

Subcellular fractionation (including MAM). Subcellular fractionation of nuclei, mitochondria, ER, MAM and cytosol from HEK293T cells was performed following the previously described protocols ${ }^{32,33}$. Cells were homogenized in isolation buffer ( $225 \mathrm{mM}$ mannitol, $75 \mathrm{mM}$ sucrose, $0.1 \mathrm{mM}$ EGTA, $30 \mathrm{mM}$ Tris-HCl pH 7.4) until $90 \%$ of cells were broken. Then, the homogenate was centrifuged at $600 \times g$ for $10 \mathrm{~min}$ three times to clarify the supernatant. The pellet (nuclear fraction) was washed three times with isolation buffer and resuspended in RIPA buffer. Collected supernatant was centrifuged for $15 \mathrm{~min}$ at $7000 \times g$ for obtaining crude mitochondria. The crude mitochondria were washed with isolation buffer, and $10 \%$ were resuspended in RIPA buffer, the other $90 \%$ were used to isolate pure mitochondria and MAM (see below). The collected supernatant was centrifuged at $20,000 \times g$ for $30 \mathrm{~min}$ to remove the plasma membrane. Then, the supernatant was centrifuged at $100,000 \times g$ for $1 \mathrm{~h}$ and the pellet was resuspended for the ER fraction and the supernatant was kept for the cytosolic fraction. For pure mitochondria and MAM fraction, the crude mitochondria pellet was resuspended in $2 \mathrm{~mL}$ MRB buffer (250 mM mannitol, $5 \mathrm{mM}$ HEPES $\mathrm{pH} 7.4,0.5 \mathrm{mM}$ EGTA), and the fraction was added on the top of $30 \%$ percoll medium $(225 \mathrm{mM}$ mannitol, $25 \mathrm{mM}$ HEPES pH 7.4, $1 \mathrm{mM}$ EGTA, 30\% percoll (v/v)) in an ultracentrifuge tube. 
Centrifugation was performed at $95,000 \times g$ for $30 \mathrm{~min}$. The bottom layer band was diluted with 10 volumes of MRB buffer and centrifuged at $6300 \times g$ for $15 \mathrm{~min}$ twice, the pellet was then saved as pure mitochondria. The upper layer band was diluted with 10 volumes of MRB buffer and centrifuged at $6300 \times g$ for $15 \mathrm{~min}$, the supernatant was collected and centrifuged again at $100,000 \times g$ for $1 \mathrm{~h}$ and the pellet was collected as MAM fraction.

Proteinase K protection assay. Proteinase $\mathrm{K}$ protection assay was performed as previously described ${ }^{18}$. Briefly, mitochondria were isolated from HEK293T cells expressing PIGBOS-FLAG and then equally divided into six samples. Samples were resuspended and incubated on ice for $30 \mathrm{~min}$ in isolation buffer $(225 \mathrm{mM}$ mannitol, $75 \mathrm{mM}$ sucrose, $50 \mathrm{mM}$ HEPES pH 7.5), $2 \mathrm{mM}$ HEPES (pH 7.5) or $2 \mathrm{mM}$ HEPES $+0.3 \%(\mathrm{v} / \mathrm{v})$ Triton X-100 (two samples for each condition). Then samples were treated with $0.5 \mu \mathrm{L}$ of proteinase K (New England Biolabs P8107S) on ice for $30 \mathrm{~min}$ (one sample for each condition). The reaction was inactivated by adding PMSF to a final concentration of $1 \mathrm{mM}$. The resulting samples were precipitated with $30 \%(\mathrm{v} / \mathrm{v}) \mathrm{TCA}$, and the pellet was washed with cold acetone and resuspended in SDS loading buffer. Protein levels were visualized by Western blotting using indicated antibodies.

APEX labeling in live cells. Biotin-phenol labeling in live cells was performed as previously described ${ }^{52}$. Briefly, PIGBOS-APEX fusion proteins or APEX control were transiently transfected into HEK293T cells using Lipofectamine 2000 Twenty-four-hours post-transfection, cell culture medium was changed to fresh growth medium containing $500 \mu \mathrm{M}$ biotin-tyramide (CDX-B0270, Adipogen). After 30 min incubation at $37^{\circ} \mathrm{C}, \mathrm{H}_{2} \mathrm{O}_{2}$ was added to each plate at a final concentration of $1 \mathrm{mM}$ and the plates were gently agitated for $1 \mathrm{~min}$. Cells were then washed three times with quenching solution $(5 \mathrm{mM}$ Trolox, $10 \mathrm{mM}$ sodium azide and $10 \mathrm{mM}$ sodium ascorbate in PBS) and the pellet was collected by centrifugation at $1000 \times g$ for $5 \mathrm{~min}$.

Immunoprecipitations. Constructs as indicated and corresponding controls were transfected into a 10-cm dish of HEK293T cells using Lipofectamine 2000 according to manufacturer's protocol. Forty-eight-hours post-transfection, cells were harvested and lysed in lysis buffer $(50 \mathrm{mM}$ Tris $\mathrm{pH} 7.4,150 \mathrm{mM} \mathrm{NaCl}, 1 \%$ Triton X-100) supplemented with Roche complete protease inhibitor cocktail tablet and $1 \mathrm{mM}$ PMSF. Cells were lysed on ice for $20 \mathrm{~min}$ followed by centrifugation at $10,000 \times g$ for $10 \mathrm{~min}$ at $4^{\circ} \mathrm{C}$ to remove cell debris. Cell lysates were added to prewashed mouse IgG agarose beads (Sigma A0919) and rotated at $4{ }^{\circ} \mathrm{C}$ for $1 \mathrm{~h}$. The supernatants were collected and added to pre-washed anti-FLAG M2 Affinity Gel (Sigma, A2220) or anti-HA agarose beads (Sigma, A2095). The suspensions were rotated at $4{ }^{\circ} \mathrm{C}$ overnight and washed four times with $1 \times$ TBST. Bound proteins were eluted with $3 \times$ FLAG peptide (Sigma, F4799) or HA peptide (Sigma, I2149) at $4^{\circ} \mathrm{C}$ for $1 \mathrm{~h}$. In some experiments, bound proteins were eluted by adding SDS loading buffer and boiled at $95^{\circ} \mathrm{C}$ for $10 \mathrm{~min}$. The eluents were then separated by SDS-PAGE and analyzed by Western blotting using indicated antibodies.

Flow cytometry analysis of PIGBOS-CLCC1 interaction. HEK293T cells were cotransfected with CLCC1-GFP(1-10)-HA and PIGBOS-3 $\times$ GFP11-FLAG (or PIGBOS- $\Delta$ C-3 $\times$ GFP11-FLAG). Seventy-two hours after transfection, cells were washed once by PBS and resuspended in FACS buffer (PBS + 0.3\% BSA) before analyzed by BD FACSCanto II system. To measure PIGBOS-CLCC1 interaction during ER stress, $2 \mu \mathrm{g} / \mathrm{ml}$ of TM (Tocris Bioscience, \#3516) or $400 \mathrm{nM}$ of TG (Tocris Bioscience, \#1138) was added to cell culture media as indicated before flow cytometry analysis. To assess the effect of ER and mitochondrial contacts, HEK293T cells were transfected with VAPB/PTPIP51 or pcDNA as mock control. Twenty-four hours after transfection, cells were transfected with CLCC1-GFP(110)-HA and PIGBOS-3 $\times$ GFP11-FLAG. GFP signals were measured $48 \mathrm{~h}$ after transfection. Flow cytometry data were analyzed by FlowJo V10 software.

Generation of PIGBOS-KO cells. PIGBOS-KO HEK293 and U2OS cells were generated according to the protocol described ${ }^{53}$. Briefly, cells were co-transfected with two CRISPR-Cas9 constructs with sgRNAs targeting at different PIGBOS gene loci. Twenty-four hours after transfection, GFP and mCherry double positive cells were isolated by FACS and single cells were sorted in a 96-well plate. PIGBOS-KO efficiency of single colonies was assessed by genotyping PCR, sequencing and Western blots. sgRNA and genotyping PCR primers are listed in Supplementary Table 5 .

Electron microscopy and analysis. Cells were cultured in 10-cm dishes and fixed with a solution of $2 \%$ paraformaldehyde and $2.5 \%$ glutaraldehyde in $0.1 \mathrm{M}$ sodium cacodylate buffer with $2 \mathrm{mM}$ calcium chloride at $\mathrm{pH} 7.4$ for 15 minutes at room temperature (RT) followed by overnight fixation at $4{ }^{\circ} \mathrm{C}$. Fixative was replaced with $0.1 \mathrm{M}$ sodium cacodylate buffer ( $\mathrm{pH} 7.4$ ), and fixed cells were then carefully detached using an angled piece of Teflon held in a hemostat. Ribbons of cells were gently transferred into Eppendorf tubes. All samples were washed three times with $0.1 \mathrm{M}$ sodium cacodylate buffer and then post-fixed in a solution of $2 \%$ osmium tetroxide reduced with $1.5 \%$ potassium ferrocyanide in $0.1 \mathrm{M}$ sodium cacodylate buffer at room temperature for $40 \mathrm{~min}$. All samples were gently pelleted between all steps. Samples were washed three times with distilled water and stained with $1 \%$ aqueous uranyl acetate for $40 \mathrm{~min}$. The pellets were dehydrated in graded steps of ethanol before infiltration with Eponate 12 resin. Once the samples were fully infiltrated, they were centrifuged for 25 minutes at $8000 \times g$ and polymerized at $60^{\circ} \mathrm{C}$ for $48 \mathrm{~h}$. Seventy-nanometers sections were cut with a Leica UC7 ultramicrotome using a diamond knife (Diatome). Images were acquired with a Zeiss Libra 120 EF-TEM with a $2 \mathrm{~K} \mathrm{CCD} \mathrm{camera} \mathrm{with} 1 \mathrm{~nm}$ pixel size. To minimize bias in measurements, all EM images were acquired, segmented, and analyzed in a blinded fashion. Only cells with intact plasma membrane profiles were selected for imaging by the blinded technician.

After the images were acquired, the MOM and nearest ER membrane surfaces were manually segmented using the TrakEM2 plugin in the Fiji/ImageJ software environment ${ }^{54}$. For ER-MOM contact measurements, we used custom Python software to generate dilated boundaries of the MOM up to $30 \mathrm{~nm}$ away from the MOM in increments of $1 \mathrm{~nm}$. The software then measured the total length of ER $\left(L_{i}\right)$ within each MOM dilation boundary $i=1, \ldots, 30$. The amount of ER surface within the region between two dilated boundaries was calculated as $l_{i}=L_{i}-L_{i-1}$. The length $\mathrm{L}$ of the total ER-MOM contact was calculated as $L=\sum_{i=1}^{i=30} l_{i}$. The average ER-MOM distance D was then calculated as the product of the contact length $l_{i}$ and the corresponding dilation distance $i$, divided by the total length $\mathrm{L}$ (Eq. 1). The ER-MOM contact coefficient ERMICC ${ }^{36}$ was calculated as the contact length $L$ divided by the product of the mitochondrial perimeter $P$ and average distance $D$ (Eq. 2). The p-value was calculated using the two tailed unpaired t-test.

$$
\begin{gathered}
D=\frac{\sum_{i=1}^{i=30} i \times l_{i}}{\sum_{i=1}^{i=30} l_{\mathrm{i}}} \\
\text { ERMICC }=\frac{L}{P \times D}
\end{gathered}
$$

XBP-1 mRNA splicing assay. XBP-1 mRNA splicing assay was performed as previously described ${ }^{55}$. In brief, total RNA was extracted using PureLink RNA mini kit (Life Technologies, 12183025) and reverse transcribed to cDNA using QuantiTect Reverse Transcription kit (Qiagen, 205313). PCR primers 5'-CGGAAGC CAAGGGGAATGAAG-3' and 5'-GGATATCAGACTCTGAATC-3' encompassing the spliced sequences in XBP-1 mRNA were used for the RT-PCR amplification with Phusion HSII polymerase (Thermo, F565). RT-PCR products were separated by electrophoresis on a $2.5 \%$ agarose gel and visualized by ethidium bromide staining. GAPDH was used as the loading control with primers $5^{\prime}$-CATGTTCC AATATGATTCCACC-3' and 5'-CTCCACGACGTACTCAGCG-3'.

ATF6 luciferase assay. HEK293 cells were plated in a 6-well plate. The second day, cells were transfected with PIGBOS siRNA or non-targeting negative siRNA as indicated using Lipofectamine RNAi MAX. Meanwhile, cells were also transfected with PIGBOS-FLAG or pcDNA3.1(+) empty vector using Lipofectamine 2000. Twenty-four hours after transfection, cells were co-transfected with p5x-ATF6-GL3 and $\beta$-galactosidase. Six hours after transfection, cells were re-seeded in a 96 well plate, which was pre-treated with $50 \mu \mathrm{g} / \mathrm{mL}$ poly-L-lysine (Sigma, P1399) and incubated overnight. The next morning, cells were treated with indicated concentrations of TM for $24 \mathrm{~h}$ before measuring luciferase activities.

Caspase-3 activity assay. PIGBOS-KD, PIGBOS-KO, and control U2OS cells were treated with indicated concentrations of TM or TG for $27 \mathrm{~h}$. Caspase-3 activities were measured using EnzChekTM Caspase-3 Assay Kit (Life Technologies, E13183). Meanwhile, total cell lysate of each condition was analyzed by Western blotting using cleaved Caspase- 3 and cleaved PARP antibodies.

Cell viability (MTT) assay. U2OS cells were transfected with PIGBOS siRNA and negative control non-targeting siRNA using Lipofectamine RNAiMAX. Forty-eight hours after transfection, cells were treated with indicated concentration of TG for $48 \mathrm{~h}$ before viability measurement by the MTT assay method. Briefly, 3-(4,5dimethylthiazol-2-yl)-2,5-diphenyltetrazolium bromide (MTT, EMD Millipore, 475989) was dissolved in PBS at a concentration of $5 \mathrm{mg} / \mathrm{mL}(10 \times)$ and then $1: 10$ diluted in DMEM $+10 \%$ FBS without phenol red (1x). Cell culture medium was replaced with $1 \times$ MTT solution, and cells were incubated at $37^{\circ} \mathrm{C}$ for $4 \mathrm{~h}$. The medium was then aspirated, and DMSO was added to dissolve the insoluble formazan product. Absorbance at $570 \mathrm{~nm}$ was measured using a BioTek Synergy H5 microplate reader.

Structure analysis for PIGBOS microprotein. PIGBOS structure was analyzed by inputting the PIGBOS microprotein sequence into the TMDOCK server ${ }^{43}$ (https:// membranome.org/tmdock). The predicted structure was illustrated by PyMol.

\section{Data availability}

The data supporting the findings are available within the article and Supplementary Information. RNA-Seq and Ribosome profiling data for PIGBOS (Supplementary Fig. 1a) are analyzed from a study that will be published separately, and the data have been 
deposited into Gene Expression Ominbus database with accession number GSE125218 (https://www.ncbi.nlm.nih.gov/geo/query/acc.cgi?acc=GSE125218). The MS data for PIGBOS-FLAG immunoprecipitation have been deposited to the ProteomeXchange Consortium via the PRIDE partner repository with the dataset identifier PXD014890. All other data are available from the authors upon reasonable request. The source data underlying Figs. 4a, d, e, 5b, 6c-e, 6g and Supplementary Figs. 13b, d, 14a, b, 15c, d, 16a, b, 17a, c, d, 18a, b and 20a-c are provided as a Source Data file.

Received: 25 June 2019; Accepted: 13 September 2019; Published online: 25 October 2019

\section{References}

1. Saghatelian, A. \& Couso, J. P. Discovery and characterization of smORFencoded bioactive polypeptides. Nat. Chem. Biol. 11, 909-916 (2015).

2. Couso, J. P. \& Patraquim, P. Classification and function of small open reading frames. Nat. Rev. Mol. Cell Biol. 18, 575-589 (2017).

3. Starck, S. R. et al. Translation from the $5^{\prime}$ untranslated region shapes the integrated stress response. Science 351, aad3867 (2016).

4. Slavoff, S. A. et al. Peptidomic discovery of short open reading frame-encoded peptides in human cells. Nat. Chem. Biol. 9, 59-64 (2013).

5. Bazzini, A. A. et al. Identification of small ORFs in vertebrates using ribosome footprinting and evolutionary conservation. EMBO J. 33, 981-993 (2014).

6. Anderson, D. M. et al. A micropeptide encoded by a putative long noncoding RNA regulates muscle performance. Cell 160, 595-606 (2015).

7. Zhang, Q. et al. The microprotein Minion controls cell fusion and muscle formation. Nat. Commun. 8, 15664 (2017).

8. Arnoult, N. et al. Regulation of DNA repair pathway choice in S and G2 phases by the NHEJ inhibitor CYREN. Nature 549, 548 (2017).

9. Walter, P. \& Ron, D. The unfolded protein response: from stress pathway to homeostatic regulation. Science 334, 1081-1086 (2011).

10. Ron, D. \& Walter, P. Signal integration in the endoplasmic reticulum unfolded protein response. Nat. Rev. Mol. Cell Biol. 8, 519-529 (2007).

11. Malhotra, J. D. \& Kaufman, R. J. ER stress and its functional link to mitochondria: role in cell survival and death. Cold Spring Harb. Perspect. Biol. 3, a004424 (2011).

12. Hetz, C. \& Papa, F. R. The unfolded protein response and cell fate control. Mol. Cell 69, 169-181 (2018).

13. Hetz, C., Chevet, E. \& Oakes, S. A. Proteostasis control by the unfolded protein response. Nat. Cell Biol. 17, 829-838 (2015).

14. Wang, S. \& Kaufman, R. J. The impact of the unfolded protein response on human disease. J. Cell. Biol. 197, 857-867 (2012).

15. Hetz, C. \& Saxena, S. ER stress and the unfolded protein response in neurodegeneration. Nat. Rev. Neurol. 13, 477-491 (2017).

16. Lin, M. F., Jungreis, I. \& Kellis, M. PhyloCSF: a comparative genomics method to distinguish protein coding and non-coding regions. Bioinformatics 27, i275-i282 (2011)

17. Krogh, A., Larsson, B., von Heijne, G. \& Sonnhammer, E. L. Predicting transmembrane protein topology with a hidden Markov model: application to complete genomes. J. Mol. Biol. 305, 567-580 (2001).

18. Aerts, L., Craessaerts, K., De Strooper, B. \& Morais, V. A. PINK1 kinase catalytic activity is regulated by phosphorylation on serines 228 and $402 . J$. Biol. Chem. 290, 2798-2811 (2015).

19. Dimmer, K. S. et al. LETM1, deleted in Wolf-Hirschhorn syndrome is required for normal mitochondrial morphology and cellular viability. Hum. Mol. Genet. 17, 201-214 (2008).

20. Kamiyama, D. et al. Versatile protein tagging in cells with split fluorescent protein. Nat. Commun. 7, 11046 (2016).

21. Chakrabarti, R. et al. INF2-mediated actin polymerization at the ER stimulates mitochondrial calcium uptake, inner membrane constriction, and division. J. Cell. Biol. 217, 251-268 (2018).

22. Walther, D. M. \& Rapaport, D. Biogenesis of mitochondrial outer membrane proteins. Biochim. Biophys. Acta 1793, 42-51 (2009).

23. D'Lima, N. G. et al. A human microprotein that interacts with the mRNA decapping complex. Nat. Chem. Biol. 13, 174 (2017).

24. Choi, H. et al. SAINT: probabilistic scoring of affinity purification-mass spectrometry data. Nat. Methods 8, 70-73 (2011).

25. Mellacheruvu, D. et al. The CRAPome: a contaminant repository for affinity purification-mass spectrometry data. Nat. Methods 10, 730-736 (2013).

26. Hung, V. et al. Proteomic mapping of cytosol-facing outer mitochondrial and ER membranes in living human cells by proximity biotinylation. eLife $\mathbf{6}$, e24463 (2017)

27. Jia, Y., Jucius, T. J., Cook, S. A. \& Ackerman, S. L. Loss of Clcc1 results in ER stress, misfolded protein accumulation, and neurodegeneration. J. Neurosci. 35, 3001-3009 (2015).
28. Nagasawa, M., Kanzaki, M., Iino, Y., Morishita, Y. \& Kojima, I. Identification of a novel chloride channel expressed in the endoplasmic reticulum, golgi apparatus, and nucleus. J. Biol. Chem. 276, 20413-20418 (2001).

29. Feng, S. et al. Improved split fluorescent proteins for endogenous protein labeling. Nat. Commun. 8, 370 (2017).

30. Vance, J. E. MAM (mitochondria-associated membranes) in mammalian cells: lipids and beyond. Biochim. Biophys. Acta 1841, 595-609 (2014).

31. van Vliet, A. R., Verfaillie, T. \& Agostinis, P. New functions of mitochondria associated membranes in cellular signaling. Biochim. Biophys. Acta 1843, 2253-2262 (2014).

32. Wieckowski, M. R., Giorgi, C., Lebiedzinska, M., Duszynski, J. \& Pinton, P. Isolation of mitochondria-associated membranes and mitochondria from animal tissues and cells. Nat. Protoc. 4, 1582 (2009).

33. Hirabayashi, Y. et al. ER-mitochondria tethering by PDZD8 regulates $\mathrm{Ca} 2+$ dynamics in mammalian neurons. Science 358, 623-630 (2017).

34. Kornmann, B. The molecular hug between the ER and the mitochondria. Curr. Opin. Cell Biol. 25, 443-448 (2013).

35. Stoica, R. et al. ER-mitochondria associations are regulated by the VAPBPTPIP51 interaction and are disrupted by ALS/FTD-associated TDP-43. Nat. Commun. 5, 3996 (2014).

36. Naon, D. et al. Critical reappraisal confirms that Mitofusin 2 is an endoplasmic reticulum-mitochondria tether. Proc. Natl Acad. Sci. USA 113, 11249-11254 (2016).

37. Wang, Y. et al. Activation of ATF6 and an ATF6 DNA binding site by the endoplasmic reticulum stress response. J. Biol. Chem. 275, 27013-27020 (2000).

38. Munch, C. The different axes of the mammalian mitochondrial unfolded protein response. BMC Biol. 16, 81 (2018).

39. Qureshi, M. A., Haynes, C. M. \& Pellegrino, M. W. The mitochondrial unfolded protein response: signaling from the powerhouse. J. Biol. Chem. 292, 13500-13506 (2017).

40. Munch, C. \& Harper, J. W. Mitochondrial unfolded protein response controls matrix pre-RNA processing and translation. Nature 534, 710-713 (2016).

41. Bernstein, S. H. et al. The mitochondrial ATP-dependent Lon protease: a novel target in lymphoma death mediated by the synthetic triterpenoid CDDO and its derivatives. Blood 119, 3321-3329 (2012).

42. Szczesna-Skorupa, E., Chen, C. D., Liu, H. \& Kemper, B. Gene expression changes associated with the endoplasmic reticulum stress response induced by microsomal cytochrome p450 overproduction. J. Biol. Chem. 279, 13953-13961 (2004).

43. Lomize, A. L. \& Pogozheva, I. D. TMDOCK: an energy-based method for modeling alpha-helical dimers in membranes. J. Mol. Biol. 429, 390-398 (2017).

44. Muñoz, J. et al. Mfn2 modulates the UPR and mitochondrial function via repression of PERK. EMBO J. 32, 2348-2361 (2013).

45. de Brito, O. M. \& Scorrano, L. Mitofusin 2 tethers endoplasmic reticulum to mitochondria. Nature 456, 605 (2008).

46. Stein, C. S. et al. Mitoregulin: a lncRNA-encoded microprotein that supports mitochondrial supercomplexes and respiratory efficiency. Cell Rep. 23, 3710-3720 (2018)

47. Oakes, S. A. \& Papa, F. R. The role of endoplasmic reticulum stress in human pathology. Annu. Rev. Pathol. 10, 173-194 (2015).

48. He, L., Diedrich, J., Chu, Y. Y. \& Yates, J. R. 3rd Extracting accurate precursor information for tandem mass spectra by rawconverter. Anal. Chem. 87, 11361-11367 (2015)

49. Xu, T. et al. ProLuCID: an improved SEQUEST-like algorithm with enhanced sensitivity and specificity. J. Proteom. 129, 16-24 (2015).

50. Tabb, D. L., McDonald, W. H. \& Yates, J. R. 3rd DTASelect and Contrast tools for assembling and comparing protein identifications from shotgun proteomics. J. Proteome Res. 1, 21-26 (2002).

51. Ma, J. et al. Improved identification and analysis of small open reading frame encoded polypeptides. Anal. Chem. 88, 3967-3975 (2016).

52. Chu, Q. et al. Identification of microprotein-protein interactions via APEX tagging. Biochemistry 56, 3299-3306 (2017).

53. Ran, F. A. et al. Genome engineering using the CRISPR-Cas9 system. Nat. Protoc. 8, 2281-2308 (2013).

54. Cardona, A. et al. TrakEM2 software for neural circuit reconstruction. PLoS One. 7, e38011 (2012)

55. Lisbona, F. et al. BAX inhibitor-1 is a negative regulator of the ER stress sensor IRE1alpha. Mol. Cell 33, 679-691 (2009).

\section{Acknowledgements}

We thank members in the Saghatelian lab, Luke Wiseman and his lab (The Scripps Research Institute) for experimental advice and helpful discussions. This research was supported by NIH/NIGMS (R01GM102491, A.S.), the Leona M. and Harry B. Helmsley Charitable Trust grant (A.S.), Dr. Frederick Paulsen Chair/Ferring Pharmaceuticals (A.S.), the George E. Hewitt Foundation for medical research (Q.C.), the Anderson Foundation (Q.C.), NIH F32 fellowship (GM123685, T.F.M.) and the Pioneer Fellowship 
(D.T.). Imaging work was supported by the Waitt Advanced Biophotonics Core Facility of the Salk Institute with funding from NIH-NCI CCSG: P30 014195, NINDS Neuroscience Core Grant: NS072031 and the Waitt Foundation. Proteomics studies were supported by the Mass Spectrometry Core of the Salk Institute with funding from the NIH with an NCI Cancer Center Support Grant P30 (CA014195).

\section{Author contributions}

Q.C., U.M. and A.S. conceived the project, designed the experiments and wrote the manuscript. Q.C., T.F.M., C.J.D., D.T. and T.C. performed experiments and analyzed data. J.M.V. generated rat and human PIGBOS antibodies, S.W.N. and L.A. performed electron microscopy experiments and analyzed the data, J.K.D. performed proteomics experiments, A.K. generated PIGBOS-KO HEK293 cells, T.Z. contributed to imaging experiments. All authors discussed the results and edited the manuscript.

\section{Competing interests}

The authors declare no competing interests.

\section{Additional information}

Supplementary information is available for this paper at https://doi.org/10.1038/s41467019-12816-z.

Correspondence and requests for materials should be addressed to U.M. or A.S.
Reprints and permission information is available at http://www.nature.com/reprints

Peer Review Information Nature Communications thanks the anonymous reviewers for their contribution to the peer review of this work.

Publisher's note Springer Nature remains neutral with regard to jurisdictional claims in published maps and institutional affiliations.

(c) Open Access This article is licensed under a Creative Commons Attribution 4.0 International License, which permits use, sharing, adaptation, distribution and reproduction in any medium or format, as long as you give appropriate credit to the original author(s) and the source, provide a link to the Creative Commons license, and indicate if changes were made. The images or other third party material in this article are included in the article's Creative Commons license, unless indicated otherwise in a credit line to the material. If material is not included in the article's Creative Commons license and your intended use is not permitted by statutory regulation or exceeds the permitted use, you will need to obtain permission directly from the copyright holder. To view a copy of this license, visit http://creativecommons.org/ licenses/by/4.0/.

(C) The Author(s) 2019 